\title{
ESTILO DE VIDA Y SÍNDROME METABÓLICO EN UNA MUESTRA DE PACIENTES DE UNA UNIDAD DE ATENCIÓN PRIMARIA
}

\author{
Nadia Isela González-Mosco', Patricia Teresa Reyes-Gabino², María Valeria Jiménez-Báez³ \\ Juan Carlos Espinoza-Rodríguez ${ }^{4}$ L Luis Sandoval-Jurad $0^{3^{*}}$ \\ 'Médico familiar; ${ }^{2}$ Coordinador clínico de Educación e Investigación en Salud. UMF N. ${ }^{0}$ 16, IMSS, Cancún; ${ }^{3}$ Coordinación auxiliar médica \\ de Investigación en Salud, IMSS; ${ }^{4}$ Profesor titular de la residencia de Medicina Familiar, UMF N. ${ }^{0}$ 16, IMSS, Cancún, Miembro del Colegio \\ de Medicina Familiar de Quintana Roo., México
}

RESUMEN: Objetivo: Determinar el estilo de vida en relación con los criterios para síndrome metabólico en una muestra de pacientes de una unidad de atención primaria en Cancún, México. Metodología: Estudio transversal en pacientes mayores de 20 años a quienes se les otorgaron cuestionarios autoaplicables con información sociodemográfica y estilo de vida mediante el instrumento FANTASTIC. Se consideró síndrome metabólico cuando se cumplieron dos o más criterios establecidos por el Adult Treatment Panel III (ATP III). Los valores de los parámetros bioquímicos fueron obtenidos del expediente electrónico; se realizó estadística descriptiva mediante el software SPSS 21. Resultados: Se incluyeron 145 pacientes, el 69.0\% de los cuales eran mujeres. La presencia de síndrome metabólico fue del $38.6 \%$ y el estilo de vida que predominó fue «bueno». Al evaluar el estilo de vida en estos pacientes se observó que la mitad de la población (50.3\%) refirió un estilo de vida «bueno». De igual manera, la mitad de los hombres y de las mujeres presentaron este mismo estilo de vida. Conclusiones: La muestra de estudio presentó una elevada frecuencia de síndrome metabólico; sin embargo, más de la mitad refirió un estilo de vida «bueno». De igual manera la mitad de los hombres y de las mujeres presentaron este mismo estilo de vida.

Palabras clave: Estilo de vida. Síndrome metabólico. Factores de riesgo cardiovascular. Atención primaria.

\section{LIFESTYLE AND METABOLIC SYNDROME IN A PATIENT SAMPLE FROM A PRIMARY CARE UNIT}

ABSTRACT: Objective: Determine the lifestyle with regard to metabolic syndrome criteria in a patient sample from a primary care unit in Cancun, Mexico. Methodology: Cross-sectional study in patients greater than 20 years of age who were provided with self-applied questionnaires to obtain sociodemographic and lifestyle information using the FANTASTIC instrument. Metabolic syndrome was considered when two or more criteria were met as established by the Adult Treatment Panel III (ATP III). Biochemical parameters were obtained from the electronic file; descriptive statistics were performed with the SPSS v 21 program. Results: A total of 145 patients were included, of which $69.0 \%$ were women. The presence of metabolic syndrome was $38.6 \%$ and a "good" lifestyle predominated. When evaluating lifestyle in these patients, it was found that half (50.3\%) referred a "good" lifestyle. According to gender, half of the men and women had the same lifestyle. Conclusions: The study sample presented a high frequency of metabolic syndrome; however, more than half expressed a "good" lifestyle. An equal percentage of men and women presented the same lifestyle. (Rev Mex Med Fam. 2018;5:55-61) Corresponding author: Luis Sandoval-Jurado, sanju.luis@gmail

Key words: Lifestyle. Metabolic syndrome. Cardiovascular risk factors. Primary care.

\section{Correspondencia:}

*Luis Sandoval-Jurado

Av. Politécnico, S/N, entre Tepic y Kinic

Región 509

C.P. 77533, Cancún, Roo., México

E-mail:sanju.luis@gmail.com 


\section{INTRODUCCIÓN}

El estilo de vida se define como el conjunto de hábitos y el comportamiento de respuesta al día a día ocupándose a través del proceso de socialización y constantemente reinterpretado y probado a lo largo del ciclo de vida en diferentes situaciones sociales ${ }^{1}$. Un estilo de vida saludable se refiere al que ofrece, preserva y aumenta el nivel de salud y bienestar ${ }^{2}$.

La Organización Mundial de la Salud (OMS) refiere que el estilo de vida está influenciado por los hábitos, actitudes, conductas, tradiciones, actividades y decisiones de una persona o un grupo de personas en relación con las diversas circunstancias en las que se desarrollan ${ }^{3}$. Es uno de los principales determinantes de los problemas de salud en países en vías de desarrollo ${ }^{4}$.

A nivel mundial, más de un tercio de las muertes son producto de estilos de vida no saludables y la causa principal de desarrollar enfermedades cardiovasculares y cronicodegenerativas ${ }^{5}$.

Los dominios que componen el estilo de vida incluyen comportamiento y preferencias relacionadas con el tipo de dieta, la nutrición, la actividad física, el consumo de alcohol, el tabaquismo y/o drogadicción, la responsabilidad de la salud, las actividades recreativas, las relaciones interpersonales, la práctica sexual, el trabajo y los patrones de actividades de carrera ${ }^{6}$.

La OMS ha estimado que 8 de los 10 factores de riesgo que conducen a la muerte de la mayoría de la gente en todo el mundo están relacionados con el estilo de vida, lo que representa el $49.9 \%$ de todas las muertes ${ }^{7}$. De éstas, las enfermedades cardiovasculares son las principales causas de muerte en el mundo, con una mortalidad de $12.9 \mathrm{mi}$ llones de personas en $2010^{8}$ y un estimado de más de 23.3 millones para el año $2030^{9}$.

Los múltiples factores de riesgo modificables asociados con las enfermedades cardiovasculares y todas las causas de mortalidad en la población general son desafíos permanentes para los tomadores de decisiones de atención primaria ${ }^{10}$. Estos factores se agrupan y clasifican en fisiológicos, bioquímicos, clínicos y metabólicos, con valores de referencia establecidos para el control de los mismos; pero su incremento indica la presencia de riesgo para enfermedad cardiovascular y mortalidad ${ }^{11}$. A este conjunto se le denominó síndrome metabólico y, de acuerdo a las guías para el tratamiento de las dislipemias en el adulto, ATP III lo identifica como un factor de riesgo múltiple para enfermedad cardiovascular ${ }^{12}$.

El cuestionario FANTASTIC fue diseñado para permitir que la persona pueda medir su estilo de vida en la población general; es una traducción y adaptación de un instrumento desarrollado por Wilson y Ciliska, del Departamento de Medicina Familiar de la Universidad de McMaster, en Ontario. Este cuestionario explora diversos campos de bienestar psicosocial, es de rápida cumplimentación y contempla los dominios referidos por la OMS para la evaluación del estilo de vida. Consta de 25 reactivos y 9 dominios, con un a de Cronbach de $0.73^{13}$ a 0.80 y un rango de 0.367 a 0.891 en el análisis por ítem. Además, este cuestionario presenta cuatro opciones de respuesta, con un valor numérico de 0 a 4 para cada categoría, las cuales se califican por medio de una escala tipo Likert, con una calificación máxima de 100 puntos $^{14,15}$. Para la población mexicana el coeficiente de correlación por rangos de Spearman entre las calificaciones totales del test y el retest de la versión de cinco opciones de respuesta fue de $\mathrm{r}=0.81(\mathrm{p}=0.01)^{16}$.

El presente trabajo tuvo como objetivo determinar el estilo de vida en relación con los criterios para síndrome metabólico en una muestra de pacientes de una unidad de atención primaria en Cancún, México.

\section{MATERIAL Y MÉTODOS}

Se realizó un estudio descriptivo, transversal y observacional en una unidad de atención primaria de Cancún, México. La población participante correspondió al rango de edad de 20 a 66 años. 
A cada paciente se le invitó a participar en el estudio el día que acudió a su cita médica con previa información sobre el objetivo del mismo, aceptación mediante consentimiento informado y con afiliación vigente.

Los datos se obtuvieron mediante un cuestionario autoaplicado sobre factores de riesgo cardiovascular como: edad, sexo, antecedentes heredofamiliares (diabetes, hipertensión, dislipemia, cardiopatías), antecedentes personales patológicos (diabetes, hipertensión, obesidad, dislipemia, tabaquismo, alcoholismo, sedentarismo), ocupación (empleados, jubilados, ama de casa, estudiantes) y estado civil (casado, soltero, viudo, divorciado).

Para la evaluación del estilo de vida se utilizó el cuestionario FANTASTIC, cuyos puntajes se estratificaron en cinco niveles: $\leq 39$ puntos, "peligro»; 40-59 puntos, «malo»; 60-69 puntos, «regular»; 70-84 puntos, «bueno» $y \geq 85$ puntos, «excelente». Para la clasificación del síndrome metabólico se utilizaron los parámetros referidos por el ATP III: glucosa sérica en ayuno $\geq 100 \mathrm{mg} / \mathrm{dl}$, presión sistólica $\geq 130 \mathrm{mmHg}$, presión diastólica $\geq 85 \mathrm{mmHg}$, lipoproteínas de alta densidad (HDL) $<40 \mathrm{mg} / \mathrm{dl}$ en hombres y $<50 \mathrm{mg} / \mathrm{dl}$ en mujeres, triglicéridos $\geq 150 \mathrm{e}$ índice de masa corporal (IMC) $>30 \mathrm{~kg} / \mathrm{m}^{2}$. Ambos instrumentos, la medición de peso, talla y de presión arterial se realizaron en un área física destinada para el estudio en la cual no existían distractores que pudieran modificar la respuesta.

La muestra se estimó mediante fórmula para obtener una proporción y estuvo constituida por 145 pacientes adscritos a la unidad médica de atención primaria que fueron seleccionados mediante un muestreo por conveniencia de los 12 consultorios de la unidad para los turnos matutino y vespertino. Los valores de glucosa y lípidos fueron obtenidos del expediente electrónico en un periodo no mayor a seis meses.

Se eliminaron las encuestas en las cuales existía una doble respuesta a la pregunta o en la cual no había respuesta para la misma. Asimismo, si en el momento de la encuesta
Tabla 1. Aspectos sociodemográficos de la población de estudio $(n=145)$

\begin{tabular}{|l|c|c|c|}
\hline & $\mathbf{n}$ & $\%$ & IC 95\% \\
\hline Situación laboral \\
\hline Desempleado & 25 & 17.2 & $11.1-23.4$ \\
\hline Con trabajo actual & 81 & 55.9 & $47.6-64.1$ \\
\hline Labores del hogar & 39 & 26.9 & $20.7-34.5$ \\
\hline Pareja actual \\
\hline Sí & 110 & 75.9 & $69.0-83.4$ \\
\hline No & 35 & 24.1 & $16.6-31.0$ \\
\hline Género & 101 & 69.7 & $23.4-37.9$ \\
\hline Femenino & 44 & 30.3 & $62.1-76.6$ \\
\hline Masculino & 37 & 25.5 & $17.9-33.1$ \\
\hline Sedentarismo & 108 & 74.5 & $66.9-82.1$ \\
\hline Sí & 30 & 20.7 & $14.5-27.6$ \\
\hline No & 115 & 79.3 & $72.4-85.5$ \\
\hline Tabaquismo & 36 & 24.8 & $18.6-32.4$ \\
\hline Sí & 109 & 75.2 & $67.6-81.4$ \\
\hline No
\end{tabular}

el paciente solicitaba asesoría sobre su estilo de vida o enfermedad por la cual recibió atención médica, se le otorgaba en un lapso no mayor a $30 \mathrm{~min}$. A cada paciente se le respetó su anonimato y los datos se manejaron con confidencialidad.

La captura y codificación de las encuestas se realizó en el software estadístico SPSS versión 21 , se determinaron las frecuencias relativas para estimar el estilo de vida y se utilizó el estadístico de chi cuadrada para identificar las diferencias entre los que obtuvieron un buen y mal estilo de vida.

El proyecto fue aprobado por el Comité Local de Investigación y Ética en Salud.

\section{RESULTADOS}

Se incluyó una muestra de 145 pacientes con un rango de edad de 20 a 60 años y una media de 46 años; predominaron las mujeres no sedentarias con trabajo remunerado que vivían en pareja (Tabla 1). 


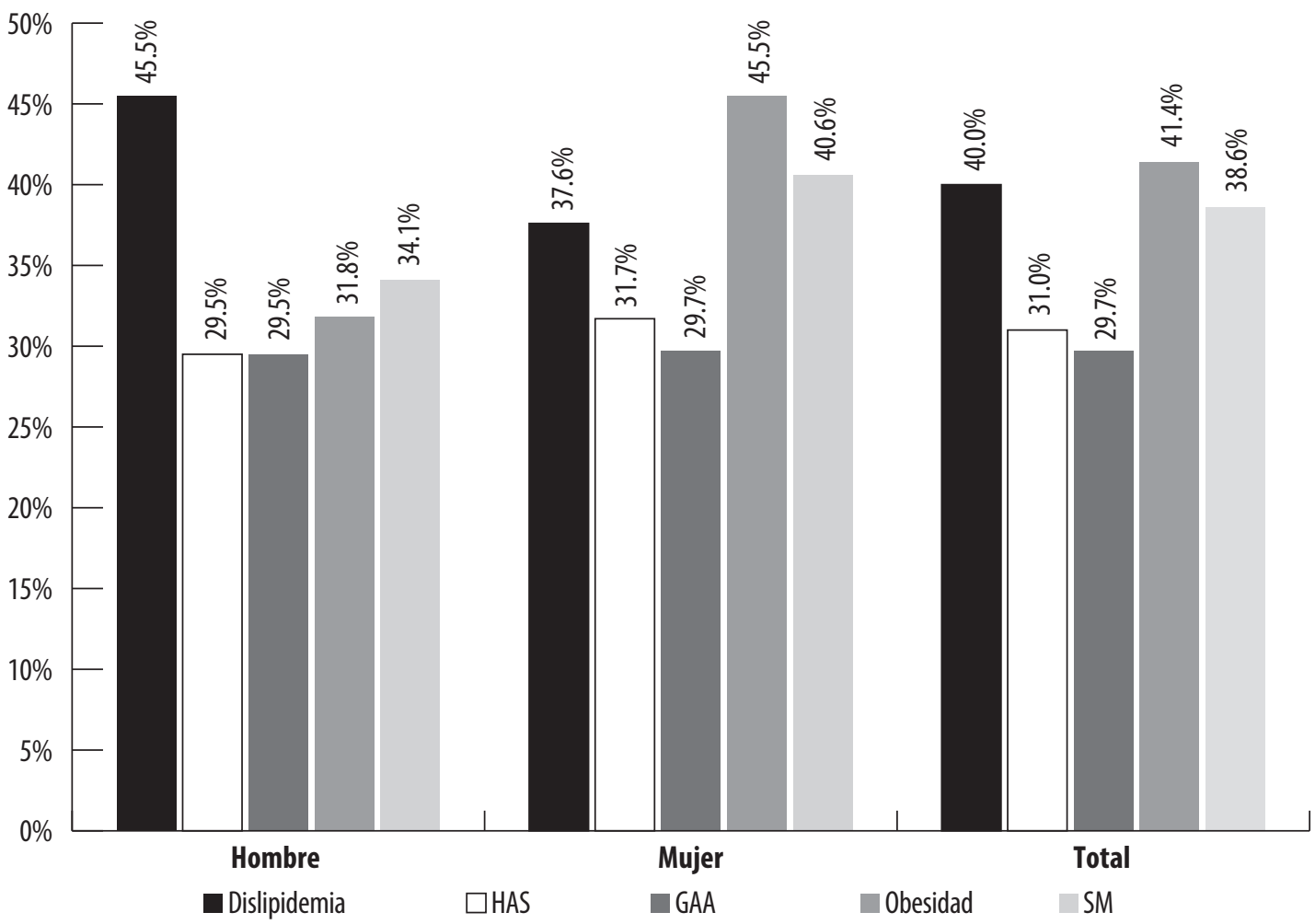

Dislipemia: definida por valores de triglicéridos mayor de $150 \mathrm{mg} / \mathrm{dl}$; HDL menor a $40 \mathrm{mg} / \mathrm{dl}$ en hombres y menor a $50 \mathrm{mg} / \mathrm{dl}$ en mujeres. Hipertensión arterial (HAS): definida por un valor de presión arterial de 130/85 mmHg o mayor y uso de fármacos antihipertensivos. Glucosa alterada en ayuno (GAA): definida por una concentración de glucosa de $100 \mathrm{mg} /$ dl o mayor 0 uso de fármacos antidiabéticos. Obesidad-0besidad abdominal: definida por un perímetro de cintura $>102 \mathrm{~cm}$ en varones $y>88 \mathrm{~cm}$ en mujeres. Se considera también un $\mathrm{IMC}>30 \mathrm{~kg} / \mathrm{m}^{2}$. Síndrome metabólico (SM): definido como la presencia de dos o más de los criterios anteriormente descritos.

Figura 1. Criterios de evaluación para síndrome metabólico $(n=145)$.

Los criterios diagnósticos para síndrome metabólico que se presentaron con mayor frecuencia en la muestra estudiada fueron dislipemia (40.0\%) y obesidad $(41.4 \%)$; en relación con el sexo, los hombres mostraron mayor frecuencia para dislipemia y las mujeres para obesidad, con un $45.5 \%$ para ambos criterios. El $38.6 \%$ de los pacientes presentaron síndrome metabólico (Fig. 1).

Al evaluar el estilo de vida de los participantes, la mitad refirió tener un estilo de vida «bueno»; al estimar la frecuencia en función del sexo, dicho estilo de vida estuvo presente en la mitad de los hombres y las mujeres. Asimismo, un estilo de vida en "peligro» fue observado en el $1.0 \%$ de las mujeres (Fig. 2).

En lo referente al estilo de vida y la presencia de síndrome metabólico, se encontró que la mitad de los pacientes que cumplieron dos o más criterios para síndrome metabólico presentaron un estilo de vida «bueno». Sin embargo, al evaluar el estilo de vida en relación con cada criterio por separado, se observó que un $20.9 \%$ de los pacientes con glucosa alterada en ayuno o diagnóstico de diabetes refirieron un estilo de vida «malo» (Tabla 2).

Al evaluar el estilo de vida también se consideraron los factores sociodemográficos, y se observó que más de la mitad de los pacientes sin pareja actual y de los desempleados reportaron un estilo de vida «bueno» (Tabla 3 ).

\section{DISCUSIÓN}

Los estilos de vida están ligados a problemas importantes de salud, los cuales se relacionan con factores de riesgo que en su mayoría pueden ser modificables. 


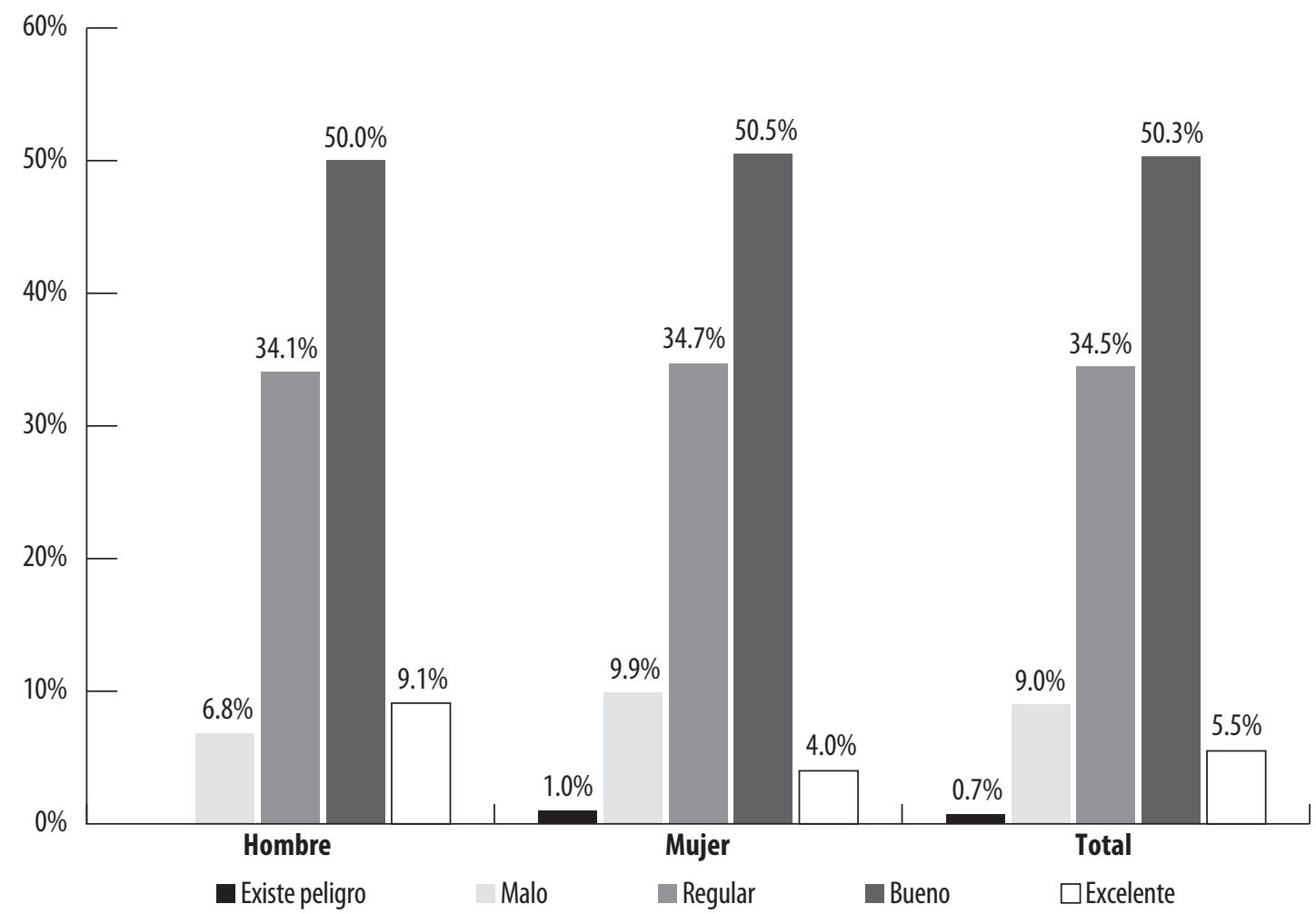

Figura 2. Estilo de vida en relación con el sexo $(n=145)$.

Tabla 2. Estilo de vida en relación con los criterios para síndrome metabólico $(n=145)$

\begin{tabular}{|c|c|c|c|c|c|c|}
\hline & Excelente & Bueno & Regular & Malo & Existe peligro & $p^{*}$ \\
\hline Dislipemia $^{\dagger}$ & $\begin{array}{c}4 \\
(6.9 \%) \\
\end{array}$ & $\begin{array}{c}30 \\
(51.7 \%) \\
\end{array}$ & $\begin{array}{c}16 \\
(27.6 \%) \\
\end{array}$ & $\begin{array}{c}8 \\
(12.5 \%) \\
\end{array}$ & $\begin{array}{c}1 \\
(1.3 \%) \\
\end{array}$ & NS \\
\hline HAS $^{\ddagger}$ & $\begin{array}{c}2 \\
(4.4 \%) \\
\end{array}$ & $\begin{array}{c}27 \\
(60.0 \%) \\
\end{array}$ & $\begin{array}{c}11 \\
(24.4 \%) \\
\end{array}$ & $\begin{array}{c}5 \\
(11.1 \%) \\
\end{array}$ & - & NS \\
\hline $\mathrm{GAA}^{\S}$ & $\begin{array}{c}3 \\
(7.0 \%)\end{array}$ & $\begin{array}{c}17 \\
(39.5 \%)\end{array}$ & $\begin{array}{c}14 \\
(32.6 \%)\end{array}$ & $\begin{array}{c}9 \\
(20.9 \%)\end{array}$ & - & 0.018 \\
\hline "Obesidad & $\begin{array}{c}3 \\
(5.0 \%)\end{array}$ & $\begin{array}{c}28 \\
(46.7 \%)\end{array}$ & $\begin{array}{c}20 \\
(33.3 \%)\end{array}$ & $\begin{array}{c}8 \\
(13.3 \%)\end{array}$ & $\begin{array}{c}1 \\
(1.7 \%)\end{array}$ & NS \\
\hline Síndrome metabólico** & $\begin{array}{c}4 \\
(7.1 \%)\end{array}$ & $\begin{array}{c}28 \\
(50.0 \%)\end{array}$ & $\begin{array}{c}16 \\
(28.6 \%)\end{array}$ & $\begin{array}{c}8 \\
(14.3 \%)\end{array}$ & - & NS \\
\hline
\end{tabular}

NS: No significativo $(p>0.05) 6.9$.

"Chi cuadrada.

'Dislipemia: definida por valores de triglicéridos mayor de $150 \mathrm{mg} / \mathrm{dl}$; HDL menor a $40 \mathrm{mg} / \mathrm{dl}$ en hombres y menor a $50 \mathrm{mg} / \mathrm{dl}$ en mujeres. *Hipertensión arterial (HAS): definida por un valor de presión arterial de $130 / 85 \mathrm{mmHg}$ o mayor y uso de fármacos antihipertensivos.

${ }^{\S} G$ lucosa alterada en ayuno (GAA): definida por una concentración de glucosa de $100 \mathrm{mg} / \mathrm{dl}$ o mayor 0 uso de fármacos antidiabéticos.

'Obesidad abdominal: definida por un perímetro de cintura $>102 \mathrm{~cm}$ en varones y $>88 \mathrm{~cm}$ en mujeres. Se considera también un IMC $>30 \mathrm{~kg} / \mathrm{m}^{2}$.

"Síndrome metabólico: definido como la presencia de dos o más de los criterios anteriormente descritos.

De acuerdo a la Encuesta Nacional de Salud y Nutrición 2016 (ENSANUT 2016) se menciona que la población adulta mexicana tiene: una prevalencia de sobrepeso y obesidad en un $72.5 \%$, siendo mayor en las mujeres, con un $87.7 \%$; hipercolesterolemia, con un 28\%; hipertensión arterial, con un $25.5 \%$, y diabetes mellitus, con un $9.4 \%{ }^{17}$. Este mismo comportamiento se presentó en el estudio, en el que la mayor 
Tabla 3. Estilo de vida en relación con las características sociales de los pacientes $(n=145)$

\begin{tabular}{|c|c|c|c|c|c|c|}
\hline & Excelente & Bueno & Regular & Malo & Existe peligro & $\mathbf{p}^{*}$ \\
\hline \multicolumn{7}{|l|}{ Pareja actual } \\
\hline Sí & $\begin{array}{c}8 \\
(7.3 \%) \\
\end{array}$ & $\begin{array}{c}53 \\
(48.2 \%) \\
\end{array}$ & $\begin{array}{c}39 \\
(35.5 \%) \\
\end{array}$ & $\begin{array}{c}10 \\
(9.1 \%) \\
\end{array}$ & - & \multirow[t]{2}{*}{ NS } \\
\hline No & - & $\begin{array}{c}20 \\
(57.1 \%)\end{array}$ & $\begin{array}{c}11 \\
(31.4 \%)\end{array}$ & $\begin{array}{c}3 \\
(8.6 \%)\end{array}$ & $\begin{array}{c}1 \\
(2.9 \%)\end{array}$ & \\
\hline \multicolumn{7}{|l|}{ Situación laboral actual } \\
\hline Desempleado & - & $\begin{array}{c}13 \\
(52.0 \%)\end{array}$ & $\begin{array}{c}11 \\
(44.0 \%)\end{array}$ & $\begin{array}{c}1 \\
(4.0 \%)\end{array}$ & - & \multirow[t]{3}{*}{ NS } \\
\hline Con trabajo actual & $\begin{array}{c}4 \\
(4.9 \%)\end{array}$ & $\begin{array}{c}42 \\
(51.9 \%)\end{array}$ & $\begin{array}{c}26 \\
(32.1 \%)\end{array}$ & $\begin{array}{c}8 \\
(9.9 \%)\end{array}$ & $\begin{array}{c}1 \\
(1.2 \%)\end{array}$ & \\
\hline Labores del hogar & $\begin{array}{c}4 \\
(10.3 \%)\end{array}$ & $\begin{array}{c}18 \\
(46.2 \%)\end{array}$ & $\begin{array}{c}13 \\
(33.3 \%)\end{array}$ & $\begin{array}{c}4 \\
(10.3 \%)\end{array}$ & & \\
\hline
\end{tabular}

NS: No significativo $(p>0.05)$.

*Chi cuadrada.

frecuencia fue para obesidad y con predominio en el sexo femenino; sin embargo, la prevalencia para dislipemia fue mayor a la referida en la ENSANUT 2016.

Abdi, et al. ${ }^{18}$ evaluaron el estilo de vida en empleados del sector público y observaron que el $61.7 \%$ presentaba un estilo de vida «bueno» en la mayoría de los reactivos evaluados, con una frecuencia mayor para las mujeres. Por el contario, en el presente estudio el estilo de vida «bueno» tuvo una frecuencia menor (50.3\%). Asimismo, los valores para hombres y mujeres fueron similares.

En Brasil, Lucas Pacheco, et al. ${ }^{19}$ evaluaron el estilo de vida en estudiantes, y encontraron una prevalencia del $5.3 \%$ para un estilo de vida "malo», a diferencia de lo obtenido en el presente estudio, en el cual el estilo de vida «malo» fue reportado sólo por el $9.0 \%$ de los participantes.

Otro estudio realizado en Colombia por Ramírez-Vélez encontró que más de la mitad de los hombres y mujeres presentaban un estilo de vida «bueno»; de igual manera se observó una correlación con el IMC ${ }^{20}$. En relación con el IMC y el estilo de vida, se observó en el presente estudio que casi la mitad (46.7\%) de los pacientes con obesidad mencionaron tener un «buen» estilo de vida.
Es importante mencionar que en la población estudiada, incluso con presencia de síndrome metabólico, el estilo de vida que predominó fue «bueno». Dos de cada $10 \mathrm{pa}$ cientes de la muestra presentan factores de riesgo modificables como sedentarismo, tabaquismo y alcoholismo; sin embargo, la mitad de la población refirió un estilo de vida «bueno», lo que puede considerarse como una adaptación a la presencia de estos factores de riesgo y, por consecuencia, no se perciben como una alteración en su estilo de vida. Entre las limitantes es importante considerar que el cuestionario FANTASTIC, de acuerdo a la validación para la población mexicana, mostró dominios con limitantes en consistencia interna y poca correlación con parámetros metabólicos.

En la aplicación de este cuestionario destaca el posible sesgo del paciente, debido a que tiende a modificar su respuesta en adecuación a lo que el personal de salud espera, lo cual se acentúa más en aquellos pacientes que han sido partícipes de acciones educativas o preventivas para la modificación de su estilo de vida.

\section{CONFLICTO DE INTERESES}

No existe conflicto de interés por parte de los autores previamente referidos. 


\section{BIBLIOGRAFÍA}

1. Guerrero LR, León A. Estilo de vida y salud. Educere. 2010; 14(48):13-9.

2. Grimaldo Muchotrigo MP. Estilo de vida saludable en estudiantes de posgrado de Ciencias de la Salud. Psicología y Salud. 2013;22(1):75-87.

3. Rondanelli IR, Rondanelli SR. Estilo de vida y enfermedad cardiovascular en el hombre. Revista Médica Clínica Las Condes. 2014;25(1):69-77.

4. Organización Mundial de la Salud. Estrategia mundial sobre régimen alimentario, actividad física y salud. ¿Por qué son importantes el sobrepeso y la obesidad infantiles? Disponible en: http://www.who.int/dietphysicalactivity/childhood_consequences/es/

5. Klein S, Sheard NF, Pi-Sunyer X, Daly A, Wylie-Rosett J, Kulkarn $\mathrm{K}$, et al. Weight Management Through Lifestyle Modification for the Prevention and Management of Type 2 Diabetes: Rationale and Strategies A statement of the American Diabetes Association, the North American Association for the Study of Obesity, and the American Society for Clinical Nutrition. Am J Clin Nutr. 2004;80(2):257-63.

6. Cerecero P, Hernández B, Aguirre D, Valdés R, Huitron G, et al. Estilos de vida asociados al riesgo cardiovascular global en trabajadores universitarios del Estado de México. Salud Pública de México. 2009;51(6):465-73.

7. Triviño LP, Dosman VA, Uribe YL, Agredo ZR, Jerez VM, Robinson $\mathrm{RV}$, et al. Estudio del estilo de vida y su relación con factores de riesgo de síndrome metabólico en adultos de mediana edad. Acta Médica Colombiana. 2009;34(4):159-63.

8. Detección y estratificación de factores de riesgo cardiovascular. México: Secretaría de Salud; 2010.

9. Fortino MA, Modini LB, Zurbriggen A, Folonier D. 1995-2010: Estudio comparativo de factores de riesgo cardiovascular en jóvenes universitarios. Rev Fed Arg Cardiol. 2012;41(4):269-76

10. Pender NM, Murdaugh C, Parson MA. Health Promotion in nursing practice. 5. ${ }^{\text {a }}$ ed. New Jersey: Pearson, Prentice Hall; 2006. p. 368.

11. Grundy SM, Cleeman JI, Daniels SR, Donato KA, Eckel RH Franklin BA, et al. Diagnosis and management of the metabolic syndrome: an American Heart Association/National Heart, Lung, and Blood Institute scientific statement. Circulation. 2005;112(17):2735-52

12. National Cholesterol Education Program (NCEP) Expert Panel on Detection, Evaluation, and Treatment of High Blood Cholesterol in Adults (Adult Treatment Panel III). Third report of the National Cholesterol Education Program (NCEP) expert panel on detection, evaluation, and treatment of high blood cholestero in adults (Adult Treatment Panel III). Final report. Circulation. 2002;106:3143-421.

13. Ramírez-Vélez R, Agredo R. Fiabilidad y validez del instrumento "Fantastico" para medir el estilo de vida en adultos colombianos. Rev Salud Publica. 2012;14(2):226-37.

14. Simpson R, Albert W, Wilson DMC, Ciliska D, Evans C. Lifestyle assessment: part 4. The Halton Health Promotion Survey. Canadian Family Physician. 1984;30:2147-55.

15. Kason Y, Ylanko VJ. FANTASTIC lifestyle assessment: part 5 measuring lifestyle in family practice. Can Fam Physician. 1984 30:2379-83.

16. López JG, Capote M. Educación continuada en consulta de enfermería especializada: la herramienta para mantener los cambios en el estilo de vida del paciente coronario. Enfermería en Cardiología. 2005;(35):13-9.

17. Velázquez-Monroy $\mathrm{O}$, Rosas Peralta $\mathrm{M}$, Lara Esqueda A, Pastelín Hernández G, Attie F, Tapia Conyer R, et al. Prevalencia e interrelación de enfermedades crónicas no transmisibles y factores de riesgo cardiovascular en México: Resultados finales de la Encuesta Nacional de Salud (ENSA) 2000. Arch Cadriol Mex. 2003;73(1):62-77.

18. Abdi J, Eftekhar H, Mahmoodi M, Shojaeizade D, Sadeghi R Estilo de vida de los empleados que trabajan en los sectores públicos Hamadan: Aplicación del Modelo transteórico. Iran Red Crescent Med J. 2015;17(2):1-7.

19. Lucas-Pacheco $R$, Santos-Silva DA, Pinheiro GA, Bianchini $O$ Petroski EL, et al. Determinantes sociodemográficos del estilo de vida en universitarios. Rev Salud Publica. 2014;6(3):382-93.

20. Ramírez-Vélez $R$, Triana-Reina HR, Carrillo HA, Ramos-Sepúlveda $J A$, Rubio F, Poches-Franco L, et al. A cross-sectional study of Colombian University students' self-perceived lifestyle. Springerplus. 2015:4:289. 